\title{
A Perceptual Computing-based Approach for Peer Assessment
}

\author{
Kok Chin Chai \\ Universiti Malaysia Sarawak \\ Kota Samarahan \\ Sarawak, Malaysia. \\ kcchai@live.com
}

\author{
Kai Meng Tay \\ Universiti Malaysia Sarawak \\ Kota Samarahan \\ Sarawak, Malaysia. \\ kmtay@feng.unimas.my
}

\begin{abstract}
Peer assessment is useful for assessing an individual's contribution in a collaborative learning task. It is commonly used in institutions of higher education as normally each class has a large number of students. Peer assessment allows students to assess their group members' contributions. It also helps the instructor to arrive at an assessment that reflects individual's effort in a collaborative learning task. In this paper, a perceptual computing (Per-C)-based peer assessment procedure is investigated. The assessment adopts vague and imprecise words in the evaluation process. Per-C is selected because it allows uncertainties of words to be considered in the decision making process. A real case study is conducted to demonstrate the effectiveness of the proposed method. The results show that the use of Per-C in peer assessment is important.
\end{abstract}

Keywords: perceptual computing, peer assessment, linguistic uncertainties, linguistic terms.

\section{Introduction}

Peer assessment is an assessment of a student's work by other students of the same class [1-5]. It is commonly used in institutions of higher education whereby each class has a large number of students, as it is difficult for the instructor to closely monitor each student's efforts in a given task [1-2]. Besides that, peer assessment provides an opportunity for students to learn from their peers [2].

Generally, there are two types of peer assessment; i.e., (1) involving students in a class to assess other students' work; (2) involving students to assess the contribution/performance of other students within the same group [6][7]. The use of these two types of peer assessment can be further classified into two: either formative or summative [6][7]. The goals of formative assessment are to monitor students' learning capabilities, gather their ongoing feedbacks and improve their learning experience [6]. On the other hand, summative assessment evaluates students' learning capabilities at the end of an instructional unit [6]. Typically, the use of peer assessment in either formative or summative form is decided by an instructor [6]. In this paper, we focus on summative assessment.
Many peer assessment methods are available in the literature [1-4] [8-11]. Traditionally, numerical grading scores are used in a way that is equivalent to psychological measurement [12]. As an example, a numerical grading scale (e.g., 1 to 5) for group members' assessment was developed in [3], whereby " 1 " was awarded for "didn't contribute", "2" for "willing but not successful", "3" for "average", " 4 " for "above average", and " 5 " for "outstanding" [3]. Even through the use of numerals in peer assessment is popular, it suffer from problems associated with psychological measurement in terms of the meaning of the numerals used (see [12] for a study on the theoretical relationship between measurement and marking).

The use of techniques related to fuzzy sets in education assessment has been widely investigated lately [2][13-17]. Fuzzy set is an efficient and effective method to represent uncertainties [13]. Comparing with methods based on numerical grading scores [1][3][4], fuzzy sets [2][13-17] provide an alternative to linguistic evaluation in which "fuzzy" words, instead of numerals, are used during the assessment procedure.

Motivated by the success of fuzzy sets in education assessment [2][13-17], the aim of this paper is to examine the effectiveness of perceptual computing (Per-C) [18-22] in peer assessment. While Per-C has been applied to decision making problems [18-22], its use in peer assessment is still new. Per-C is chosen for this study owing to its ability to handle subjectivity, vagueness, imprecision, and uncertainty while achieving tractability and robustness in modelling human decision-making behaviours [18-22]. Comparing with type-1 fuzzy models [2][13-17], Per-C adopts interval type-2 fuzzy sets (IT2FSs) in tackling a decision making problem. Comparing with type-1 fuzzy sets, IT2FSs are able to provide more flexibility in preserving and processing uncertainties in decision-making tasks [18].

The general structure of Per-C is depicted in Figure 1. It consists of three components [18-21], i.e., an encoder, a computing-with-words (CWW) engine, and a decoder. Linguistic perceptions or words from human operators are converted into IT2FSs through the encoder. The CWW 